KEK-TH-503

TIT/HEP-357

hep-th/9612115

December 1996

\title{
A Large-N Reduced Model as Superstring
}

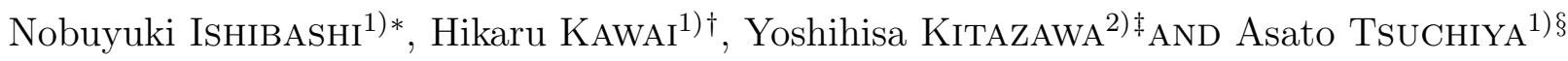 \\ 1) National Laboratory for High Energy Physics (KEK), \\ Tsukuba, Ibaraki 305, Japan \\ 2) Department of Physics, Tokyo Institute of Technology, \\ Oh-okayama, Meguro-ku, Tokyo 152, Japan
}

\begin{abstract}
A matrix model which has the manifest ten-dimensional $N=2$ super Poincare invariance is proposed. Interactions between BPS-saturated states are analyzed to show that massless spectrum is the same as that of type IIB string theory. It is conjectured that the large- $\mathrm{N}$ reduced model of ten-dimensional super Yang-Mills theory can be regarded as a constructive definition of this model and therefore is equivalent to superstring theory.
\end{abstract}

\footnotetext{
* e-mail address : ishibash@theory.kek.jp

$\dagger$ e-mail address : kawaih@theory.kek.jp

$\ddagger$ e-mail address : kitazawa@th.phys.titech.ac.jp

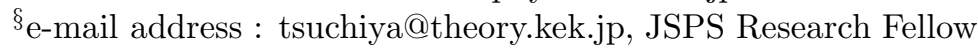




\section{Introduction}

A number of recent developments on the nonperturbative aspects of string theory have revealed the fact that various superstring theories can be equivalent even though their original definitions look totally different [1, 2, 3]. It seems that the universality class of superstring theory is unique so that all the consistent quantum theories of gravity are equivalent. Contrary to these remarkable developments, we still have not reached the final stage in understanding the string dynamics, and we definitely need a non-perturbative or constructive definition of string theory.

In this paper, as a candidate for such constructive definition, we would like to propose the large- $\mathrm{N}$ reduced model of ten-dimensional super Yang-Mills theory «1. If we assume the existence of its continuum limit we can present several evidences which indicate that it is equivalent to superstring theory and thus gives its constructive definition. Our work is motivated by the recent work of Banks, Fischler, Shenker and Susskind [0] in which they propose a matrix model quantum mechanics. While their model is relevant to a possible nonperturbative definition of M-theory [2], our model is directly related to type IIB superstring.

In section 2 we propose a matrix model which looks like the Green-Schwarz action of type IIB string [6] in the Schild gauge [7]. This matrix model has the manifest Lorentz invariance and $N=2$ space-time supersymmetry. Therefore if the theory is totally well-defined it will be a constructive definition of string theory. However, this theory has an infrared divergence and we have to remove it by some renormalization, which we discuss in section 4 .

In section 3 we consider the one-loop effective action of the model and examine the interactions between the BPS-saturated objects, and show that the massless spectrum of the theory is indeed consistent with that of type IIB theory. This is another evidence that the matrix model considered here is equivalent to string theory.

In section 4 we discuss the renormalization of the matrix model and show that it can be regarded as the continuum limit of the large- $\mathrm{N}$ reduced model of ten-dimensional super Yang-Mills theory. Here the old idea of the large-N reduction of the degrees of freedom plays a rather important role. In contrast to the non-supersymmetric case, because of the supersymmetry, the $U(1)^{d}$ symmetry is not spontaneously broken but preserved marginally even in the weak coupling limit. Therefore in this limit the eigenvalues of the gauge fields become free, and they play the role of the space-time coordinates. It turns out that the 
space-time is dynamically generated as a collective coordinate of this model.

Appendix is devoted to show the technical details of the one-loop calculations.

\section{Relations between Green-Schwarz superstring and a matrix model}

In this section, we examine the path integral of type IIB string theory in the Schild gauge and propose a matrix model which can be regarded as its rigorous definition.

We start with the covariant Green-Schwarz action of type IIB superstring expressed in the Nambu-Goto form [6]:

$$
\begin{aligned}
S_{G S}=-T \int d^{2} \sigma \quad( & \sqrt{-\frac{1}{2} \Sigma^{2}}+i \epsilon^{a b} \partial_{a} X^{\mu}\left(\bar{\theta}^{1} \Gamma_{\mu} \partial_{b} \theta^{1}+\bar{\theta}^{2} \Gamma_{\mu} \partial_{b} \theta^{2}\right) \\
& \left.+\epsilon^{a b} \bar{\theta}^{1} \Gamma^{\mu} \partial_{a} \theta^{1} \bar{\theta}^{2} \Gamma_{\mu} \partial_{b} \theta^{2}\right) .
\end{aligned}
$$

Here $\theta^{1}$ and $\theta^{2}$ are Majorana-Weyl spinors in ten dimensions having the same chiralities. $\Sigma^{\mu \nu}$ and $\Pi_{a}^{\mu}$ are defined by

$$
\begin{aligned}
\Sigma^{\mu \nu} & =\epsilon^{a b} \Pi_{a}^{\mu} \Pi_{b}^{\nu}, \\
\Pi_{a}^{\mu} & =\partial_{a} X^{\mu}-i \bar{\theta}^{1} \Gamma^{\mu} \partial_{a} \theta^{1}+i \bar{\theta}^{2} \Gamma^{\mu} \partial_{a} \theta^{2} .
\end{aligned}
$$

Note that we have applied an analytic continuaton in the path integral, $\theta^{2} \rightarrow i \theta^{2}$, so that the signs of the terms including $\theta^{2}$ are opposite to those of the ordinary ones. (See the footnote below.) This system possesses the $N=2$ space-time supersymmetry

$$
\begin{aligned}
\delta_{S U S Y} \theta^{1} & =\epsilon^{1}, \\
\delta_{S U S Y} \theta^{2} & =\epsilon^{2}, \\
\delta_{S U S Y} X^{\mu} & =i \bar{\epsilon}^{1} \Gamma^{\mu} \theta^{1}-i \bar{\epsilon}^{2} \Gamma^{\mu} \theta^{2},
\end{aligned}
$$

and the $\kappa$-symmetry

$$
\begin{aligned}
\delta_{\kappa} \theta^{1} & =\alpha^{1}, \\
\delta_{\kappa} \theta^{2} & =\alpha^{2}, \\
\delta_{\kappa} X^{\mu} & =i \bar{\theta}^{1} \Gamma^{\mu} \alpha^{1}-i \bar{\theta}^{2} \Gamma^{\mu} \alpha^{2},
\end{aligned}
$$


where

$$
\begin{aligned}
\alpha^{1} & =(1+\tilde{\Gamma}) \kappa^{1} \\
\alpha^{2} & =(1-\tilde{\Gamma}) \kappa^{2} \\
\tilde{\Gamma} & =\frac{1}{2 \sqrt{-\frac{1}{2} \Sigma^{2}}} \Sigma_{\mu \nu} \Gamma^{\mu \nu}
\end{aligned}
$$

Since $\kappa^{1}$ and $\kappa^{2}$ are local fermionic parameters and $\tilde{\Gamma}^{2}=1$, half of the degrees of freedom of $\theta^{1}$ and $\theta^{2}$ are redundant, and the $\kappa$-symmetry can be gauge-fixed by imposing the condition $\theta^{1}=\theta^{2}=\psi$. This condition is compatible with Lorentz symmetry because $\theta^{1}$ and $\theta^{2}$ have the same chirality in type IIB string theory. This gauge-fixing leads to the action

$$
\tilde{S}_{G S}=-T \int d^{2} \sigma\left(\sqrt{-\frac{1}{2} \sigma^{2}}+2 i \epsilon^{a b} \partial_{a} X^{\mu} \bar{\psi} \Gamma_{\mu} \partial_{b} \psi\right),
$$

where

$$
\sigma^{\mu \nu}=\epsilon^{a b} \partial_{a} X^{\mu} \partial_{b} X^{\nu}
$$

The action $\tilde{S}_{G S}$ is still invariant under the $N=2$ supersymmetry, provided one modifies the transformation law (2.3) by mixing it with the $\kappa$-symmetry transformation so that the gauge fixing condition $\theta^{1}=\theta^{2}$ is preserved. The new transformation law becomes,

$$
\begin{aligned}
\delta \theta^{1} & =\delta_{S U S Y} \theta^{1}+\delta_{\kappa} \theta^{1} \\
\delta \theta^{2} & =\delta_{S U S Y} \theta^{2}+\delta_{\kappa} \theta^{2} \\
\delta X^{\mu} & =\delta_{S U S Y} X^{\mu}+\delta_{\kappa} X^{\mu},
\end{aligned}
$$

where we choose $\kappa^{1}$ and $\kappa^{2}$ as

$$
\begin{aligned}
\kappa^{1} & =\frac{-\epsilon^{1}+\epsilon^{2}}{2}, \\
\kappa^{2} & =\frac{\epsilon^{1}-\epsilon^{2}}{2}
\end{aligned}
$$

\footnotetext{
" Here we comment on the analytic continuation. For an example, we consider the quantum fluctuations of $\psi$ around the static solution $X^{0}=\tau, X^{1}=\sigma$ and $X^{i}=0(i \geq 2)$. Then the action (2.6) results in $2 i T \int d^{2} \sigma\left(\psi_{R}^{T}\left(\partial_{\tau}+\partial_{\sigma}\right) \psi_{R}-\psi_{L}^{T}\left(\partial_{\tau}-\partial_{\sigma}\right) \psi_{L}\right)$. By redefining $\psi_{L}$ as $\psi_{L} \rightarrow-i \psi_{L}$, we obtain the ordinary fermion action of type IIB superstring. We need this prescription in quantizing the theory in the hamiltonian formalism. This comes from the analytic continuation, $\theta^{2} \rightarrow i \theta^{2}$, applied in the path integral quantization of (2.1) because the gauge fixing leads to $\psi_{R}=\theta_{R}^{1}$ and $\psi_{L}=\theta_{L}^{2}$ in this case. Therefore what we have done is to analytically continue the degrees of freedom which is gauged away. As we see below in the (2.32), we also need this kind of prescription naturally in defining the supercharges to obtain the correct algebra of $N=2$ supersymmetry.
} 
so that $\delta \theta^{1}=\delta \theta^{2}$. By introducing $\xi$ and $\epsilon$ as

$$
\begin{aligned}
& \xi=\frac{\epsilon^{1}+\epsilon^{2}}{2}, \\
& \epsilon=\frac{\epsilon^{1}-\epsilon^{2}}{2},
\end{aligned}
$$

we can express the $N=2$ supersymmetry of $\tilde{S}_{G S}$ as follows:

$$
\begin{aligned}
\delta^{(1)} \psi & =-\frac{1}{2 \sqrt{-\frac{1}{2} \sigma^{2}}} \sigma_{\mu \nu} \Gamma^{\mu \nu} \epsilon, \\
\delta^{(1)} X^{\mu} & =4 i \bar{\epsilon} \Gamma^{\mu} \psi,
\end{aligned}
$$

and

$$
\begin{aligned}
\delta^{(2)} \psi & =\xi, \\
\delta^{(2)} X^{\mu} & =0 .
\end{aligned}
$$

In order to rewrite $\tilde{S}_{G S}$ in the Schild gauge, we first introduce the Poisson bracket as

$$
\{X, Y\} \equiv \frac{1}{\sqrt{g}} \epsilon^{a b} \partial_{a} X \partial_{b} Y .
$$

Here $\sqrt{g}$ is a positive definite scalar density defined on the world sheet which can be identified with $\sqrt{\operatorname{det}\left(g_{a b}\right)}$ made from a worldsheet metric $g_{a b}$. Using this, we write a Schild-type action [7] as

$$
S_{\text {Schild }}=\int d^{2} \sigma\left[\sqrt{g} \alpha\left(\frac{1}{4}\left\{X^{\mu}, X^{\nu}\right\}^{2}-\frac{i}{2} \bar{\psi} \Gamma^{\mu}\left\{X^{\mu}, \psi\right\}\right)+\beta \sqrt{g}\right] .
$$

As was shown by Schild some time ago, this action is equivalent to the original action $\tilde{S}_{G S}$ (2.6) at least classically. Indeed, from the equation of motion for $\sqrt{g}$ in (2.14)

$$
-\frac{1}{4} \alpha \frac{1}{(\sqrt{g})^{2}}\left(\epsilon^{a b} \partial_{a} X^{\mu} \partial_{b} X^{\nu}\right)^{2}+\beta=0,
$$

we obtain

$$
\sqrt{g}=\frac{1}{2} \sqrt{\frac{\alpha}{\beta}} \sqrt{\left(\epsilon^{a b} \partial_{a} X^{\mu} \partial_{b} X^{\nu}\right)^{2}} .
$$

Then by substituting it into (2.14), we obtain

$$
\int d^{2} \sigma\left(\sqrt{\alpha \beta} \sqrt{\left(\epsilon^{a b} \partial_{a} X^{\mu} \partial_{b} X^{\nu}\right)^{2}}-\frac{i}{2} \alpha \epsilon^{a b} \partial_{a} X^{\mu} \bar{\psi} \Gamma^{\mu} \partial_{b} \psi\right),
$$

which is equivalent to $\tilde{S}_{G S}$ up to a normalization of $\psi$, which we can adjust freely. The $N=2$ supersymmetry (2.11) and (2.12) possessed by $\tilde{S}_{G S}$ manifests itself in $S_{\text {Schild }}$ as

$$
\begin{aligned}
\delta^{(1)} \psi & =-\frac{1}{2} \sigma_{\mu \nu} \Gamma^{\mu \nu} \epsilon, \\
\delta^{(1)} X^{\mu} & =i \bar{\epsilon} \Gamma^{\mu} \psi,
\end{aligned}
$$


and

$$
\begin{aligned}
\delta^{(2)} \psi & =\xi, \\
\delta^{(2)} X^{\mu} & =0 .
\end{aligned}
$$

Although it is not clear to what extent the theory in this gauge is controllable as a full quantum theory, at least formally the quantum theory is expressed as a path integral over $X^{\mu}$ and $\sqrt{g}$ :

$$
\mathcal{Z}=\int \mathcal{D} \sqrt{g} \mathcal{D} X \mathcal{D} \psi e^{-S_{\text {Schild }}}
$$

Instead of trying to give a rigorous definition of (2.20), we first try to construct a matrix model which is equivalent to (2.20) at least in the classical limit and interpret its path integral as the definition of (2.20).

In the following, we show that the system defined by 2.20 ) can be regarded as a sort of the classical limit of the system defined by

$$
Z=\sum_{n=0}^{\infty} \int d A d \psi e^{-S}
$$

and

$$
S=\alpha\left(-\frac{1}{4} \operatorname{Tr}\left[A_{\mu}, A_{\nu}\right]^{2}-\frac{1}{2} \operatorname{Tr}\left(\bar{\psi} \Gamma^{\mu}\left[A_{\mu}, \psi\right]\right)\right)+\beta \operatorname{Tr} 1 .
$$

Here $A_{\mu}$ and $\psi$ are bosonic and fermionic $n \times n$ hermitian matrices respectively. If large values of $n$ dominate in (2.21) and the dominant distributions of eigenvalues for $A_{\mu}$ and $\psi$ are smooth enough, we expect that the commutator and the trace can be replaced with the Poisson bracket and the integration, respectively:

$$
\begin{aligned}
-i[,] & \Rightarrow\{,\}, \\
T r & \Rightarrow \int d^{2} \sigma \sqrt{g} .
\end{aligned}
$$

This is the same as the ordinary correspondence between the quantum and classical mechanics. As is well known, the basic properties of the commutator and the trace

$$
\begin{aligned}
\operatorname{Tr}[X, Y] & =0 \\
\operatorname{Tr}(X[Y, Z]) & =\operatorname{Tr}(Z[X, Y]),
\end{aligned}
$$

are preserved after taking the classical limit:

$$
\begin{aligned}
\int d^{2} \sigma \sqrt{g}\{X, Y\} & =0 \\
\int d^{2} \sigma \sqrt{g} X\{Y, Z\} & =\int d^{2} \sigma \sqrt{g} Z\{X, Y\} .
\end{aligned}
$$


Now it is obvious that $S(2.22)$ becomes $S_{\text {Schild }}$ (2.14) after this replacement [8]. We also note that the sum over $n$ in (2.21) corresponds to the path integration over $\sqrt{g}$ in (2.20). Furthermore we can easily check that the $N=2$ supersymmetry (2.18) and (2.19) is directly translated into the symmetry of $S$ as

$$
\begin{aligned}
\delta^{(1)} \psi & =\frac{i}{2}\left[A_{\mu}, A_{\nu}\right] \Gamma^{\mu \nu} \epsilon, \\
\delta^{(1)} A_{\mu} & =i \bar{\epsilon} \Gamma_{\mu} \psi,
\end{aligned}
$$

and

$$
\begin{aligned}
\delta^{(2)} \psi & =\xi \\
\delta^{(2)} A_{\mu} & =0 .
\end{aligned}
$$

At this stage we can not claim that 2.21) gives a totally consistent theory that satisfies the unitarity, causality and so on. However we will see in section 4 that (2.21) can be regarded as an effective theory of a more complete theory, that is, the large- $\mathrm{N}$ reduced model of super Yang-Mills theory. Here we simply point out that the form of $S$ in (2.22) can be regarded as a naive zero volume limit of ten-dimensional super Yang-Mills theory, except for the term proportional to $\beta$. Then it is obvious that the symmetry (2.26) is nothing but the zero volume limit of $N=1$ supersymmetry of the super Yang-Mills theory. To verify that the symmetries (2.26) and (2.27) indeed form the $N=2$ supersymmetry, we examine the commutators of these transformations below. First of all we note that the action (2.22) possesses the zero volume version of the gauge symmetry,

$$
\begin{aligned}
\delta_{\text {gauge }} A_{\mu} & =i\left[A_{\mu}, \alpha\right] \\
\delta_{\text {gauge }} \psi & =i[\psi, \alpha] .
\end{aligned}
$$

Up to the above gauge symmetry and the equation of motion for $\psi$, we have the following commutation relations:

$$
\begin{aligned}
\left(\delta_{\epsilon^{1}}^{(1)} \delta_{\epsilon^{2}}^{(1)}-\delta_{\epsilon^{2}}^{(1)} \delta_{\epsilon^{1}}^{(1)}\right) \psi & =0 \\
\left(\delta_{\epsilon^{1}}^{(1)} \delta_{\epsilon^{2}}^{(1)}-\delta_{\epsilon^{2}}^{(1)} \delta_{\epsilon^{1}}^{(1)}\right) A_{\mu} & =0 .
\end{aligned}
$$

We can also easily check the following commutators:

$$
\begin{aligned}
\left(\delta_{\epsilon}^{(1)} \delta_{\xi}^{(2)}-\delta_{\xi}^{(2)} \delta_{\epsilon}^{(1)}\right) \psi & =0 \\
\left(\delta_{\epsilon}^{(1)} \delta_{\xi}^{(2)}-\delta_{\xi}^{(2)} \delta_{\epsilon}^{(1)}\right) A_{\mu} & =i \bar{\epsilon} \Gamma_{\mu} \xi
\end{aligned}
$$


and

$$
\begin{aligned}
\left(\delta_{\xi^{1}}^{(2)} \delta_{\xi^{2}}^{(2)}-\delta_{\xi^{2}}^{(2)} \delta_{\xi^{1}}^{(2)}\right) \psi & =0 \\
\left(\delta_{\xi^{1}}^{(2)} \delta_{\xi^{2}}^{(2)}-\delta_{\xi^{2}}^{(2)} \delta_{\xi^{1}}^{(2)}\right) A_{\mu} & =0
\end{aligned}
$$

If we take a linear combination of $\delta^{(1)}$ and $\delta^{(2)}$ as

$$
\begin{aligned}
& \tilde{\delta}_{(1)}=\delta^{(1)}+\delta^{(2)}, \\
& \tilde{\delta}_{(2)}=i\left(\delta^{(1)}-\delta^{(2)}\right),
\end{aligned}
$$

we obtain the $N=2$ supersymmetry algebra,

$$
\begin{aligned}
\left(\tilde{\delta}_{\epsilon}^{(1)} \tilde{\delta}_{\xi}^{(1)}-\tilde{\delta}_{\xi}^{(1)} \tilde{\delta}_{\epsilon}^{(1)}\right) \psi & =0 \\
\left(\tilde{\delta}_{\epsilon}^{(1)} \tilde{\delta}_{\xi}^{(1)}-\tilde{\delta}_{\xi}^{(1)} \tilde{\delta}_{\epsilon}^{(1)}\right) A_{\mu} & =2 i \bar{\epsilon} \Gamma_{\mu} \xi \\
\left(\tilde{\delta}_{\epsilon}^{(2)} \tilde{\delta}_{\xi}^{(2)}-\tilde{\delta}_{\xi}^{(2)} \tilde{\delta}_{\epsilon}^{(2)}\right) \psi & =0 \\
\left(\tilde{\delta}_{\epsilon}^{(2)} \tilde{\delta}_{\xi}^{(2)}-\tilde{\delta}_{\xi}^{(2)} \tilde{\delta}_{\epsilon}^{(2)}\right) A_{\mu} & =2 i \bar{\epsilon} \Gamma_{\mu} \xi \\
\left(\tilde{\delta}_{\epsilon}^{(1)} \tilde{\delta}_{\xi}^{(2)}-\tilde{\delta}_{\xi}^{(2)} \tilde{\delta}_{\epsilon}^{(1)}\right) \psi & =0 \\
\left(\tilde{\delta}_{\epsilon}^{(1)} \tilde{\delta}_{\xi}^{(2)}-\tilde{\delta}_{\xi}^{(2)} \tilde{\delta}_{\epsilon}^{(1)}\right) A_{\mu} & =0 .
\end{aligned}
$$

The system we consider here has the manifest Lorentz invariance and is probably unitary. Therefore the global $N=2$ supersymmetry (2.33) is extended to the local one inevitably since the $N=1$ supersymmetry is maximal in ten-dimensional gauge theory. If the theory admits massless spectrum, it contains gravitons. Furthermore as we discuss just below, the path integral (2.21) automatically includes not only one-string states but also multi-string states. When the matrices $A_{\mu}$ 's and $\psi$ are block-diagonal, the action is decomposed into the sum of traces for each of the blocks. Each trace results in the string action in the Schild gauge in the classical limit. Then the trace in (2.22) corresponds to the integrals over the disconnected worldsheets. Namely, each block represents a string and the theory defined by (2.21) include multi-strings states. As we will see in the next section, the path integral of the off-diagonal-blocks generate interactions between the diagonal-blocks. Because of these facts, we expect that the theory (2.21) after a slight modification discussed in section 4 possibly gives a constructive definition of the string theory. 


\section{One-loop quantum corrections around classical solu- tions}

\subsection{Classical static D-string solutions}

In this section, we consider the typical classical solutions of (2.22) which represent infinitely long static D-strings [3]. When $\psi=0$, the equation of motion of (2.14) is

$$
\left\{X^{\mu},\left\{X^{\mu}, X^{\nu}\right\}\right\}=0 .
$$

Corresponding to this, the equation of motion of (2.22) is

$$
\left[A_{\mu},\left[A_{\mu}, A_{\nu}\right]\right]=0 .
$$

We can easily construct a solution of (3.1), which represents a static D-string extending straightly in the $X^{1}$ direction:

$$
\begin{aligned}
X^{0} & =T \tau, \\
X^{1} & =\frac{L}{2 \pi} \sigma, \\
\text { other } X^{\mu} \text { s } & =0
\end{aligned}
$$

where $T$ and $L$ are large enough compactification radii and

$$
\begin{aligned}
& 0 \leq \tau \leq 1, \\
& 0 \leq \sigma \leq 2 \pi .
\end{aligned}
$$

Considering the relation between the commutator and the Poisson bracket, we obtain a solution of (3.2) corresponding to the above one as follows:

$$
\begin{aligned}
A_{0} & =\frac{T}{\sqrt{2 \pi n}} q \equiv p_{0}, \\
A_{1} & =\frac{L}{\sqrt{2 \pi n}} p \equiv p_{1}, \\
\text { other } A_{\mu} \text { 's } & =0,
\end{aligned}
$$

where $T$ and $L$ are large enough compactification radii, and $q$ and $p$ are $n \times n$ hermitian matrices having the following commutation relation and the eigenvalue distributions:

$$
[q, p]=i,
$$


and

$$
\begin{aligned}
& 0 \leq q \leq \sqrt{2 \pi n} \\
& 0 \leq p \leq \sqrt{2 \pi n} .
\end{aligned}
$$

Strictly speaking such $p$ and $q$ do not exist for finite values of $n$. For large values of $n$, however, we expect that (3.6) can be approximately satisfied, because it is nothing but the canonical commutation relation. As is well-known in the correspondence between the classical and quantum mechanics, the total area of the $p-q$ phase space is equal to $2 \pi$ multiplied by the dimension of the representation. In this sense (3.7) indicates that $p$ and $q$ are $n \times n$ matrices.

We can also construct classical solutions corresponding to two static D-strings in the same way. As was mentioned at the end of the previous section, we can obtain these solutions by considering $A_{\mu}$ 's composed of two diagonal-blocks. First, we consider two parallel D-strings, which extend infinitely in the $X^{1}$ direction and separated by distance $b$ in the $X^{2}$ direction. The solution of (3.1) representing this situation is given by

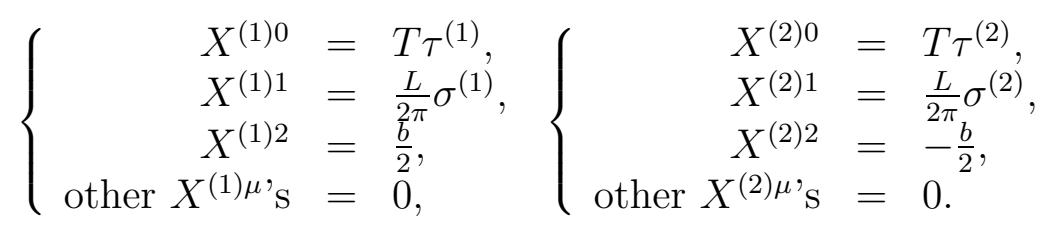

By identifying the first block with the first D-string and the second block with the second D-string, we obtain the solution of (3.2) corresponding to the above one

$$
\begin{aligned}
A_{0} & =\left(\begin{array}{cc}
\frac{T}{\sqrt{2 \pi n}} q & 0 \\
0 & \frac{T}{\sqrt{2 \pi n}} q^{\prime}
\end{array}\right) \equiv p_{0}, \\
A_{1} & =\left(\begin{array}{cc}
\frac{L}{\sqrt{2 \pi n}} p & 0 \\
0 & \frac{L}{\sqrt{2 \pi n}} p^{\prime}
\end{array}\right) \equiv p_{1}, \\
A_{2} & =\left(\begin{array}{cc}
\frac{b}{2} & 0 \\
0 & -\frac{b}{2}
\end{array}\right) \equiv p_{2}, \\
\text { other } A_{\mu}{ }^{\prime} \mathrm{s} & =0,
\end{aligned}
$$

where $q^{\prime}$ and $p^{\prime}$ satisfy the same properties as (3.6) and (3.7). Applying a unitary transformation, we can set $q^{\prime}$ equal to $q$ and $p^{\prime}$ equal to $p$. Similarly for the solution of (3.1) representing two anti-parallel D-strings

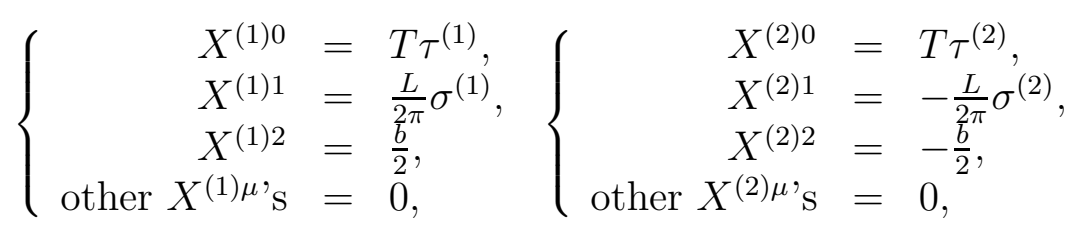


we obtain the corresponding solution of (3.2)

$$
\begin{aligned}
A_{0} & =\left(\begin{array}{cc}
\frac{T}{\sqrt{2 \pi n}} q & 0 \\
0 & \frac{T}{\sqrt{2 \pi n}} q
\end{array}\right) \equiv p_{0}, \\
A_{1} & =\left(\begin{array}{cc}
\frac{L}{\sqrt{2 \pi n}} p & 0 \\
0 & -\frac{L}{\sqrt{2 \pi n}} p
\end{array}\right) \equiv p_{1}, \\
A_{2} & =\left(\begin{array}{cc}
\frac{b}{2} & 0 \\
0 & -\frac{b}{2}
\end{array}\right) \equiv p_{2}, \\
\text { other } A_{\mu} \text { 's } & =0 .
\end{aligned}
$$

Finally, we consider a general configurations of two static straight D-strings described by

$$
\left\{\begin{array} { r l } 
{ X ^ { ( 1 ) 0 } } & { = T \tau ^ { ( 1 ) } , } \\
{ X ^ { ( 1 ) 1 } } & { = \frac { L } { 2 \pi } \sigma ^ { ( 1 ) } , } \\
{ X ^ { ( 1 ) 2 } } & { = 0 , } \\
{ X ^ { ( 1 ) 3 } } & { = \frac { b } { 2 } , } \\
{ \text { other } X ^ { ( 1 ) \mu } , \mathrm { s } } & { = 0 , }
\end{array} \quad \left\{\begin{array}{rl}
X^{(2) 0} & =T \tau^{(2)} \\
X^{(2) 1} & =\frac{L}{2 \pi} \sigma^{(2)} \cos \theta, \\
X^{(2) 2} & =\frac{L}{2 \pi} \sigma^{(2)} \sin \theta, \\
X^{(2) 3} & =-\frac{b}{2}, \\
\text { other } X^{(2) \mu}, \mathrm{s} & =0,
\end{array}\right.\right.
$$

where $b$ is the minimum distance between the two D-strings . The solution of (3.2) corresponding to the above one is

$$
\begin{aligned}
A_{0} & =\left(\begin{array}{cc}
\frac{T}{\sqrt{2 \pi n}} q & 0 \\
0 & \frac{T}{\sqrt{2 \pi n}} q
\end{array}\right) \equiv p_{0}, \\
A_{1} & =\left(\begin{array}{cc}
\frac{L}{\sqrt{2 \pi n}} p & 0 \\
0 & \frac{L}{\sqrt{2 \pi n}} p \cos \theta
\end{array}\right) \equiv p_{1}, \\
A_{2} & =\left(\begin{array}{cc}
0 & 0 \\
0 & \frac{L}{\sqrt{2 \pi n}} p \sin \theta
\end{array}\right) \equiv p_{2}, \\
A_{3} & =\left(\begin{array}{cc}
\frac{b}{2} & 0 \\
0 & -\frac{b}{2}
\end{array}\right) \equiv p_{3}, \\
\text { other } A_{\mu}^{\prime} \mathrm{s} & =0
\end{aligned}
$$

\subsection{One-loop effective action and stability of BPS-saturated states}

In appendix, we calculate the one-loop effective action $W$ around a general background $A_{\mu}=p_{\mu}$. The result is

$$
R e W=\frac{1}{2} \operatorname{Tr} \log \left(P_{\lambda}^{2} \delta_{\mu \nu}-2 i F_{\mu \nu}\right)-\frac{1}{4} \operatorname{Tr} \log \left(\left(P_{\lambda}^{2}+\frac{i}{2} F_{\mu \nu} \Gamma^{\mu \nu}\right)\left(\frac{1+\Gamma_{11}}{2}\right)\right)-\operatorname{Tr} \log \left(P_{\lambda}^{2}\right) .
$$

Here $P_{\mu}$ and $F_{\mu \nu}$ are operators acting on the space of matrices as

$$
\begin{aligned}
P_{\mu} X & =\left[p_{\mu}, X\right], \\
F_{\mu \nu} X & =\left[f_{\mu \nu}, X\right],
\end{aligned}
$$


where $f_{\mu \nu}=i\left[p_{\mu}, p_{\nu}\right]$. In (3.14), each of the three terms corresponds to the contributions from the bosons $A_{\mu}$, the fermions $\psi$ and the Fadeev-Popov ghosts, respectively.

The cases in which $f_{\mu \nu}=c-$ number $\equiv c_{\mu \nu}$ have a special meaning. These correspond to BPS-saturated backgrounds [9]. Indeed, by setting $\xi$ equal to $\pm \frac{1}{2} c_{\mu \nu} \Gamma^{\mu \nu} \epsilon$ in the $N=2$ supersymmetry (2.26) and (2.27), we obtain the relations

$$
\begin{aligned}
\left(\delta^{(1)} \mp \delta^{(2)}\right) \psi & =0, \\
\left(\delta^{(1)} \mp \delta^{(2)}\right) A_{\mu} & =0 .
\end{aligned}
$$

Namely, half of the supersymmetry is preserved in these backgrounds. Since $F_{\mu \nu}=0$ in these cases, we have

$$
R e W=\left(\frac{1}{2} \cdot 10-\frac{1}{4} \cdot 16-1\right) \operatorname{Tr} \log \left(P_{\lambda}^{2}\right)=0
$$

and

$$
\operatorname{Im} W=0
$$

as is shown in appendix, which means the one-loop quantum corrections vanish due to the supersymmetry. This is consistent with the well-known fact that the BPS-saturated states have no corrections and are stable. One of the simplest examples of the BPS-saturated states is the solution (3.5). Indeed, for this solution,

$$
\begin{aligned}
f_{01}=i\left[p_{0}, p_{1}\right] & =-\frac{T L}{2 \pi n}, \\
\text { other } f_{\mu \nu} \text { 's } & =0 .
\end{aligned}
$$

We see that an infinitely long static D-string exists stably.

More specifically we may consider the cases in which $c_{\mu \nu}=0$, that is, $\left[p_{\mu}, p_{\nu}\right]=0$. Then we can diagonalize all $p_{\mu}$ 's simultaneously. These are classical minima of the action and are often called moduli in supersymmetric gauge theories. The one-loop effective action also vanishes in these cases, and there are no interactions between the eigenvalues. Namely, the moduli space is stable even quantum mechanically, which is a manifestation of the non-renormalization theorem in supersymmetric theories. The eigenvalues tend to spread randomly and their distribution is likely to be uniform, which means that $U(1)^{d}$ symmetry is marginally preserved. Note that if we had no contributions from fermions, there would be attractive logarithmic interactions between the eigenvalues and they would concentrate on one value. This is the well-known $U(1)^{d}$ symmetry breaking in the weak coupling region of non-supersymmetric gauge theories. 


\subsection{Interactions between two static D-strings}

First we consider the solution (3.9) representing the two parallel static D-strings. Since in this case

$$
\begin{aligned}
f_{01}=i\left[p_{0}, p_{1}\right] & =-\frac{T L}{2 \pi n}, \\
\text { other } f_{\mu \nu} \text { 's } & =0,
\end{aligned}
$$

this solution represents a BPS-saturated state and the one-loop effective action is equal to zero. We obtain a consistent picture that there is no force between two parallel D-strings due to the cancellation of the gravitational force with the force mediated by the anti-symmetric tensor field. This implies the possibility of superposing BPS-saturated states.

As a preparation of the calculations of the quantum corrections to the solutions (3.11) and (3.13), we evaluate the one-loop effective action (3.14) when $\left[p_{\mu}, f_{\nu \lambda}\right]=c-$ number. In these cases $P_{\lambda}^{2}$ and $F_{\mu \nu}$ commute with each other and are simultaneously diagonalizable. We set $F_{\mu \nu}$ in the standard form

$$
F_{\mu \nu}=\left(\begin{array}{ccccc}
0 & -a_{1} & & & \\
a_{1} & 0 & & & \\
& & \ddots & & \\
& & & 0 & -a_{5} \\
& & & a_{5} & 0
\end{array}\right)
$$

and calculate each term of (3.14) as follows. The first term is

$$
\operatorname{Tr} \log \left(P_{\lambda}^{2} \delta_{\mu \nu}-2 i F_{\mu \nu}\right)=\sum_{i=1}^{5} \operatorname{Tr} \log \left(\left(P_{\lambda}^{2}\right)^{2}-4 a_{i}^{2}\right) .
$$

Considering that the eigenvalues of $\Gamma^{\mu \nu}$ are equal to $\pm i$ and that we project the system to the space in which the eigenvalue of $\Gamma_{11}=i \Gamma^{1} \Gamma^{2} \cdots \Gamma^{10}$ is equal to 1 , we evaluate the second term of $(3.14)$ as

$$
\left.\operatorname{Tr} \log \left(\left(P_{\lambda}^{2}+\frac{i}{2} F_{\mu \nu} \Gamma^{\mu \nu}\right)\right)\left(\frac{1+\Gamma_{11}}{2}\right)\right)=\sum_{s_{1}, \ldots, s_{5}= \pm 1, s_{1} \cdots s_{5}=1} \operatorname{Tr} \log \left(P_{\lambda}^{2}-\left(a_{1} s_{1}+\cdots+a_{5} s_{5}\right)\right) .
$$

From (3.14), 3.22) and (3.23), we obtain

$$
\begin{aligned}
\text { ReW }= & \frac{1}{2} \sum_{i=1}^{5} \operatorname{Tr} \log \left(1-\frac{4 a_{i}^{2}}{\left(P_{\lambda}^{2}\right)^{2}}\right) \\
& -\frac{1}{4} \sum_{s_{1}, \ldots, s_{5}= \pm 1, s_{1} \cdots s_{5}=1} \operatorname{Tr} \log \left(1-\frac{a_{1} s_{1}+\cdots+a_{5} s_{5}}{P_{\lambda}^{2}}\right) .
\end{aligned}
$$


We apply this expression to the cases we consider. Since these cases correspond to case(2) in appendix, we have $\operatorname{Im} W=0$. For the solution (3.11) corresponding to the two anti-parallel static D-strings, we have

$$
\begin{array}{r}
f_{01}=i\left[p_{0}, p_{1}\right]=-\frac{T L}{2 \pi n} \otimes \sigma^{3}, \\
\text { other } f_{\mu \nu} \text { 's }=0,
\end{array}
$$

and therefore

$$
\left[p_{\mu}, f_{\nu \lambda}\right]=0
$$

where $\sigma^{3}$ is the third component of Pauli matrices. As in (3.15) we define the adjoint operator $\Sigma^{3}$ corresponding to $1 \otimes \sigma^{3}$ as

$$
\Sigma^{3} X=\left[1 \otimes \sigma^{3}, X\right]
$$

Then we have

$$
\left[P_{0}, P_{1}\right]=i \frac{T L}{2 \pi n} \Sigma^{3}
$$

and

$$
P_{2}=\frac{b}{2} \Sigma^{3}
$$

The eigenvalues of $\Sigma^{3}$ are equal to $0,0,2$ and -2 . If the eigenvalue is equal to zero, $F_{\mu \nu}=0$ and there is no contribution to the one-loop effective action. If it is equal to \pm 2 , $P_{\lambda}^{2}$ in (3.24) behaves like a harmonic oscillator because of the commutator (3.28), whose eigenvalues are $4\left(\frac{T L}{2 \pi n}\right)\left(k+\frac{1}{2}\right)+b^{2}$. Each of these eigenvalues has $n$-fold degeneracy, because the operator $P_{\lambda}^{2}$ is acting on the space of $n \times n$ matrices. Therefore we can calculate (3.24) in this case as

$$
\begin{aligned}
W & =-n \log \prod_{k=0}^{\infty} \frac{\left(1+\frac{1}{2 k+1+\frac{b^{\prime^{2}}}{2}}\right)^{4}\left(1-\frac{1}{2 k+1+\frac{b^{\prime 2}}{2}}\right)^{4}}{\left.2 k \frac{2}{2 k+1+\frac{b^{\prime 2}}{2}}\right)\left(1-\frac{2}{2 k+1+\frac{b^{\prime 2}}{2}}\right)} \\
& =-n \log \left[\left(\frac{b^{\prime 2}}{4}\right)^{-4} \frac{\frac{b^{\prime 2}}{4}+\frac{1}{2}}{\frac{{b^{\prime 2}}^{2}}{4}-\frac{1}{2}}\left(\frac{\Gamma\left(\frac{b^{\prime 2}}{4}+\frac{1}{2}\right)}{\Gamma\left(\frac{b^{\prime 2}}{4}\right)}\right)^{8}\right],
\end{aligned}
$$

where $b^{\prime}=\sqrt{\frac{2 \pi n}{T L}} b$. This expression can be expanded with respect to $1 / b^{2}$ as

$$
W=-8 n\left(\frac{T L}{2 \pi n}\right)^{3} \frac{1}{b^{6}}+O\left(\frac{1}{b^{8}}\right) .
$$

We see that there is an interaction which is mediated by massless particles between the two anti-parallel D-strings, which should be twice as large as the gravitational interaction because of that of anti-symmetric tensor field. 
Next, we evaluate the interaction between the two generally located static D-strings described by (3.13). If we introduce $p_{1}^{\prime}$ and $p_{2}^{\prime}$ as

$$
\begin{aligned}
& p_{1}^{\prime}=\sin \frac{\theta}{2} p_{1}-\cos \frac{\theta}{2} p_{2}=\frac{L}{\sqrt{2 \pi n}} p \sin \frac{\theta}{2} \otimes \sigma^{3}, \\
& p_{2}^{\prime}=\cos \frac{\theta}{2} p_{1}+\sin \frac{\theta}{2} p_{2}=\frac{L}{\sqrt{2 \pi n}} p \cos \frac{\theta}{2} \otimes 1_{2},
\end{aligned}
$$

the background field strength is expressed as

$$
\begin{aligned}
& f_{01}^{\prime}=i\left[p_{0}, p_{1}^{\prime}\right]=-\frac{T L}{2 \pi n} \sin \frac{\theta}{2} \otimes \sigma^{3}, \\
& f_{02}^{\prime}=i\left[p_{0}, p_{2}^{\prime}\right]=-\frac{T L}{2 \pi n} \cos \frac{\theta}{2} \otimes 1_{2}, \\
& f_{12}^{\prime}=0,
\end{aligned}
$$

and hence

$$
\begin{aligned}
F_{01}^{\prime} & =-\frac{T L}{2 \pi n} \sin \frac{\theta}{2} \Sigma^{3}, \\
\text { other } F_{\mu \nu}^{\prime} ' \mathrm{~s} & =0
\end{aligned}
$$

Therefore the calculation of the one-loop effective action can be reduced to that of the two anti-parallel D-strings case, except for the nontrivial $P_{2}^{\prime}$, which commutes with the other $P_{\mu}^{\prime}$ 's. In this case the eigenvalues of $\left(P_{2}^{\prime}\right)^{2}$ are distributed uniformly from 0 to $\left(\frac{L}{\sqrt{2 \pi n}} \sqrt{2 \pi n} \cos \frac{\theta}{2}\right)^{2}$. Therefore instead of the $n$-fold degeneracy in (3.30), this time we should integrate over the eigenvalues of $P_{2}^{\prime}$. Thus for large values of $b$ we have

$$
\begin{aligned}
W & =-8 \sqrt{\frac{n}{2 \pi}} \int_{0}^{\sqrt{2 \pi n}} d p \frac{\left(\frac{T L}{2 \pi n} \sin \frac{\theta}{2}\right)^{3}}{\left(b^{2}+\left(p_{2}^{\prime}\right)^{2}\right)^{3}} \\
& =-\frac{3 \pi}{2} n\left(\frac{T L}{2 \pi n}\right)^{3} \frac{1}{L b^{5}} \sin ^{2} \frac{\theta}{2} \tan \frac{\theta}{2},
\end{aligned}
$$

where

$$
p_{2}^{\prime}=\frac{L}{\sqrt{2 \pi n}} p \cos \frac{\theta}{2} .
$$

In the following, we show that this result can be interpreted as the exchange of anti-symmetric tensor field $B_{\mu \nu}$ and graviton $G_{\mu \nu}$ between the two D-strings. In the Lorentz-type gauge, the potential created by the first D-string at distance $r$ is given in the leading order of $1 / r$ by

$$
\begin{aligned}
& B_{01}=\frac{2 \alpha^{\prime 3}}{r^{6}}, \\
& G_{00}=1-\frac{\alpha^{\prime 3}}{r^{6}}, \\
& G_{11}=1-\frac{\alpha^{\prime 3}}{r^{6}} .
\end{aligned}
$$


On the other hand, the source for the anti-symmetric tensor field and the energy momentum tensor carried by the second D-string are given by

$$
J^{01}=\frac{1}{\alpha^{\prime}} \cos \theta
$$

and

$$
\begin{aligned}
T^{00} & =\frac{1}{\alpha^{\prime}} \\
T^{11} & =\frac{1}{\alpha^{\prime}} \cos ^{2} \theta
\end{aligned}
$$

respectively. Therefore a fraction $L d \sigma$ of the second D-string located at distance $r$ from the first one possesses the potential energy

$$
2 \alpha^{\prime 2} \frac{\cos \theta}{r^{6}}-\alpha^{\prime 2} \frac{1+\cos ^{2} \theta}{r^{6}}=-4 \alpha^{\prime 2} \frac{\sin ^{4} \frac{\theta}{2}}{r^{6}}
$$

and the effective action is evaluated as

$$
-T L \int_{0}^{2 \pi} d \sigma \frac{\alpha^{\prime 2} \sin ^{4} \frac{\theta}{2}}{\left(b^{2}+L^{2} \sigma^{2} \sin ^{2} \theta\right)^{3}}=-n \frac{T L}{2 \pi n} \frac{\alpha^{\prime 2}}{b^{5} L} \sin ^{2} \frac{\theta}{2} \tan \frac{\theta}{2} .
$$

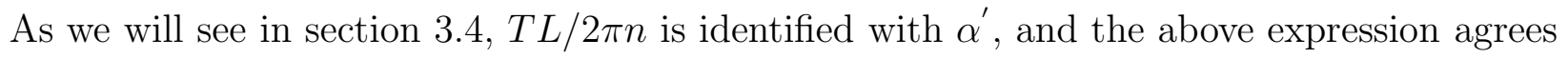
with 3.35$)$.

\subsection{Interactions between diagonal blocks and cluster property}

In this subsection, we calculate the one-loop effective action between diagonal blocks in order to identify the interactions included in the effective action with those of string theory. We consider backgrounds having a block-diagonal form:

$$
A_{\mu}=p_{\mu}=\left(\begin{array}{cccc}
p_{\mu}^{(1)} & & & \\
& p_{\mu}^{(2)} & & \\
& & p_{\mu}^{(3)} & \\
& & & \ddots
\end{array}\right)
$$

where $p_{\mu}^{(i)}(i=1,2, \cdots)$ is a $n_{i} \times n_{i}$ matrix. We may regard each $p_{\mu}^{(i)}$ as a D-object occupying some region of space-time. Here we use a term D-object to represent D-instantons, D-strings, D-3 branes, ... and their mixtures. We decompose $p_{\mu}^{(i)}$ as

$$
\begin{aligned}
p_{\mu}^{(i)} & =d_{\mu}^{(i)} 1_{n_{i}}+\tilde{p}_{\mu}^{(i)}, \\
\operatorname{Tr} \tilde{p}_{\mu}^{(i)} & =0
\end{aligned}
$$


where $d_{\mu}^{(i)}$ is a real number representing the center of mass coordinate of the $i$-th block. Here we assume that the blocks are separated far enough from each other, that is, for all $i$ and j's, $\left(d_{\mu}^{(i)}-d_{\mu}^{(j)}\right)^{2}$ 's are large.

We first introduce some notations. We denote the $(i, j)$ block of a matrix $X$ as $X^{(i, j)}$. It is clear that $P_{\mu}$ defined in 3.15 operates on each $X^{(i, j)}$ independently. In fact we have

$$
\left(P_{\mu} X\right)^{(i, j)}=\left(d_{\mu}^{(i)}-d_{\mu}^{(j)}\right) X^{(i, j)}+\tilde{p}_{\mu}^{(i)} X^{(i, j)}-X^{(i, j)} \tilde{p}_{\mu}^{(j)}
$$

We further simplify this equation by introducing notations such as

$$
\begin{aligned}
d_{\mu}^{(i, j)} X^{(i, j)} & =\left(d_{\mu}^{(i)}-d_{\mu}^{(j)}\right) X^{(i, j)} \\
P_{L \mu}^{(i, j)} X^{(i, j)} & =\tilde{p}_{\mu}^{(i)} X^{(i, j)} \\
P_{R \mu}^{(i, j)} X^{(i, j)} & =-X^{(i, j)} \tilde{p}_{\mu}^{(j)} .
\end{aligned}
$$

Note that $d_{\mu}^{(i, j)}, P_{L \mu}^{(i, j)}$ and $P_{R \mu}^{(i, j)}$ commute each other, and the operation of $P_{\mu}$ on $X^{(i, j)}$ is expressed as

$$
P_{\mu} X^{(i, j)}=\left(d_{\mu}^{(i, j)}+P_{L \mu}^{(i, j)}+P_{R \mu}^{(i, j)}\right) X^{(i, j)} .
$$

We similarly simplify the form of $F_{\mu \nu}$. The background field strength is evaluated as

$$
\begin{aligned}
& f_{\mu \nu}=\left(\begin{array}{lll}
i\left[p_{\mu}^{(1)}, p_{\nu}^{(1)}\right] & & \\
& i\left[p_{\mu}^{(2)}, p_{\nu}^{(2)}\right] & \\
& & \ddots
\end{array}\right) \\
& =\left(\begin{array}{lll}
\tilde{f}_{\mu \nu}^{(1)} & & \\
& \tilde{f}_{\mu \nu}^{(2)} & \\
& & \ddots
\end{array}\right),
\end{aligned}
$$

and we can also decompose $F_{\mu \nu}$ in the same way as (3.46):

$$
\left(F_{\mu \nu} X\right)^{(i, j)}=\left(F_{L \mu \nu}^{(i, j)}+F_{R \mu \nu}^{(i, j)}\right) X^{(i, j)},
$$

where

$$
\begin{aligned}
F_{L \mu \nu}^{(i, j)} X^{(i, j)} & =\tilde{f}_{\mu \nu}^{(i)} X^{(i, j)}, \\
F_{R \mu \nu}^{(i, j)} X^{(i, j)} & =-X^{(i, j)} \tilde{f}_{\mu \nu}^{(j)} .
\end{aligned}
$$

Since the left and right multiplication are totally independent, we have

$$
\operatorname{Tr} O=\sum_{i, j=1}^{n} \operatorname{Tr} O_{L}^{(i, j)} \operatorname{Tr} O_{R}^{(i, j)},
$$


for operators consisting of $P_{\mu}$ and $F_{\mu \nu}$.

Now we expand the general expression of the one-loop effective action (3.14) with respect to the inverse power of $d_{\mu}^{(i, j)}$ 's. We can take traces of the $\gamma$ matrices after expanding the logarithm in (3.14). Due to the supersymmetry, contributions of bosons and fermions cancel each other to the third order in $F_{\mu \nu}$, and we have,

$$
\begin{aligned}
W= & -\operatorname{Tr}\left(\frac{1}{P^{2}} F_{\mu \nu} \frac{1}{P^{2}} F_{\nu \lambda} \frac{1}{P^{2}} F_{\lambda \rho} \frac{1}{P^{2}} F_{\rho \mu}\right) \\
& -2 \operatorname{Tr}\left(\frac{1}{P^{2}} F_{\mu \nu} \frac{1}{P^{2}} F_{\lambda \rho} \frac{1}{P^{2}} F_{\mu \rho} \frac{1}{P^{2}} F_{\lambda \nu}\right) \\
& +\frac{1}{2} \operatorname{Tr}\left(\frac{1}{P^{2}} F_{\mu \nu} \frac{1}{P^{2}} F_{\mu \nu} \frac{1}{P^{2}} F_{\lambda \rho} \frac{1}{P^{2}} F_{\lambda \rho}\right) \\
& +\frac{1}{4} \operatorname{Tr}\left(\frac{1}{P^{2}} F_{\mu \nu} \frac{1}{P^{2}} F_{\lambda \rho} \frac{1}{P^{2}} F_{\mu \nu} \frac{1}{P^{2}} F_{\lambda \rho}\right)+O\left(\left(F_{\mu \nu}\right)^{5}\right) .
\end{aligned}
$$

Since as in (3.46) and (3.48) $P_{\mu}$ and $F_{\mu \nu}$ act on the $(i, j)$ blocks independently, the oneloop effective action $W$ is expressed as the sum of contributions of the $(i, j)$ blocks $W^{(i, j)}$. Therefore we may consider $W^{(i, j)}$ as the interaction between the i-th and j-th blocks. Using (3.50) and (3.51) we can easily evaluate $W^{(i, j)}$ to the leading order of $1 / \sqrt{\left(d^{(i)}-d^{(j)}\right)^{2}}$ as

$$
\begin{aligned}
W^{(i, j)}= & \frac{1}{\left(d^{(i)}-d^{(j)}\right)^{8}} \\
& \left(-\operatorname{Tr}^{(i, j)}\left(F_{\mu \nu} F_{\nu \lambda} F_{\lambda \rho} F_{\rho \mu}\right)-2 \operatorname{Tr}^{(i, j)}\left(F_{\mu \nu} F_{\lambda \rho} F_{\mu \rho} F_{\lambda \nu}\right)\right. \\
& \left.+\frac{1}{2} \operatorname{Tr}^{(i, j)}\left(F_{\mu \nu} F_{\mu \nu} F_{\lambda \rho} F_{\lambda \rho}\right)+\frac{1}{4} \operatorname{Tr}^{(i, j)}\left(F_{\mu \nu} F_{\lambda \rho} F_{\mu \nu} F_{\lambda \rho}\right)\right) \\
& +O\left(\left(1 /\left(d^{(i)}-d^{(j)}\right)^{9}\right)\right. \\
= & \frac{1}{4\left(d^{(i)}-d^{(j)}\right)^{8}} \\
& \left(-4 n_{j} \operatorname{Tr}\left(\tilde{f}_{\mu \nu}^{(i)} \tilde{f}_{\nu \lambda}^{(i)} \tilde{f}_{\lambda \rho}^{(i)} \tilde{f}_{\rho \mu}^{(i)}\right)-8 n_{j} \operatorname{Tr}\left(\tilde{f}_{\mu \nu}^{(i)} \tilde{f}_{\lambda \rho}^{(i)} \tilde{f}_{\mu \rho}^{(i)} \tilde{f}_{\lambda \nu}^{(i)}\right)\right. \\
& +2 n_{j} \operatorname{Tr}\left(\tilde{f}_{\mu \nu}^{(i)} \tilde{f}_{\mu \nu}^{(i)} \tilde{f}_{\lambda \rho}^{(i)} \tilde{f}_{\lambda \rho}^{(i)}\right)+n_{j} \operatorname{Tr}\left(\tilde{f}_{\mu \nu}^{(i)} \tilde{f}_{\lambda \rho}^{(i)} \tilde{f}_{\mu \nu}^{(i)} \tilde{f}_{\lambda \rho}^{(i)}\right) \\
& -4 n_{i} \operatorname{Tr}\left(\tilde{f}_{\mu \nu}^{(j)} \tilde{f}_{\nu \lambda}^{(j)} \tilde{f}_{\lambda \rho}^{(j)} \tilde{f}_{\rho \mu}^{(j)}\right)-8 n_{i} \operatorname{Tr}\left(\tilde{f}_{\mu \nu}^{(j)} \tilde{f}_{\lambda \rho}^{(j)} \tilde{f}_{\mu \rho}^{(j)} \tilde{f}_{\lambda \nu}^{(j)}\right) \\
& +2 n_{i} \operatorname{Tr}\left(\tilde{f}_{\mu \nu}^{(j)} \tilde{f}_{\mu \nu}^{(j)} \tilde{f}_{\lambda \rho}^{(j)} \tilde{f}_{\lambda \rho}^{(j)}\right)+n_{i} \operatorname{Tr}\left(\tilde{f}_{\mu \nu}^{(j)} \tilde{f}_{\lambda \rho}^{(j)} \tilde{f}_{\mu \nu}^{(j)} \tilde{f}_{\lambda \rho}^{(j)}\right) \\
& \left.-48 \operatorname{Tr}\left(\tilde{f}_{\mu \nu}^{(i)} \tilde{f}_{\nu \lambda}^{(i)}\right) \operatorname{Tr}\left(\tilde{f}_{\mu \rho}^{(j)} \tilde{f}_{\rho \lambda}^{(j)}\right)+6 \operatorname{Tr}\left(\tilde{f}_{\mu \nu}^{(i)} \tilde{f}_{\mu \nu}^{(i)}\right) \operatorname{Tr}\left(\tilde{f}_{\lambda \rho}^{(j)} \tilde{f}_{\lambda \rho}^{(j)}\right)\right) \\
& +O\left(\left(1 /\left(d^{(i)}-d(j)\right)^{9}\right) .\right.
\end{aligned}
$$

By observing the tensor structures of the last two terms in (3.52), we find the exchanges of massless particles corresponding to graviton and a scalar.

As we have seen, the interactions between two blocks are weaker than or equal to $1 / r^{8}$, where $r$ is the distance between two centers of mass. Therefore if D-objects are located far 
enough from each other, they can exist independently and the system possess the cluster property. This cluster property is important to the $N=2$ supersymmetry in the following sense. It is obvious that the trace parts of $A_{\mu}$ and $\psi$, or the parts proportional to identity matrix, are not included in the action (2.22), but play an essential role in the $N=2$ supersymmetry transformations. This rather puzzling situation may be resolved as follows. Due to the cluster property, the trace parts of diagonal-blocks become collective coordinates and acquire the physical meaning as the centers of mass of the D-objects. In other words, space time coordinate is generated dynamically as the trace parts. Therefore we are allowed to treat the trace parts of matrices.

\subsection{Determination of $\alpha$ and $\beta$}

In this subsection, we express the parameters $\alpha$ and $\beta$ in (2.22) in terms of physical quantities using the results of the previous subsections. Substituting one static D-string solution (3.5) into (2.22), we obtain

$$
S=\alpha \frac{1}{4}\left(\frac{T L}{2 \pi n}\right)^{2} n+\beta n
$$

By taking the variation in terms $n$, we have

$$
\frac{T L}{2 \pi n}=2 \sqrt{\frac{\beta}{\alpha}}
$$

as the equation of motion for $n$. Substituting this into (3.53), we obtain

$$
S=\frac{1}{2 \pi} \sqrt{\alpha \beta} T L
$$

This should be equal to the classical value of the Nambu-Goto action $\rho T L$, where $\rho$ is the string tension of the D-string. Therefore we have

$$
\sqrt{\alpha \beta}=2 \pi \rho
$$

We can also find the ratio of $\alpha$ and $\beta$ by examining the interaction between two D-strings. As is well-known, $\rho$ and $\kappa$ are expressed in terms of $\alpha^{\prime}$ and $g_{s}$ as

$$
\begin{aligned}
& \rho=4 \pi^{5 / 2} \frac{\alpha^{\prime}}{\kappa}, \\
& \kappa=\gamma \alpha^{\prime 2} g_{s},
\end{aligned}
$$


where $2 \kappa^{2}$ is 16 times gravitation constant and $g_{s}$ is the string coupling. In terms of these quantities the effective action between two anti-parallel static D-strings is expressed as

$$
W=-2 \rho^{2} \frac{2 \kappa^{2}}{4}\left(\frac{\pi^{4}}{3}\right)^{-1} \frac{1}{b^{6}} T L
$$

where the factor $\pi^{4} / 3$ comes from the area of $S^{7}$. Requiring that this quantity is equal to (3.31) and using (3.54), we obtain a relation,

$$
2 \sqrt{3} \pi \alpha^{\prime}=\frac{T L}{2 \pi n}=2 \sqrt{\frac{\beta}{\alpha}}
$$

From (3.56) and (3.59), $\alpha$ and $\beta$ are determined as

$$
\begin{aligned}
& \alpha=\frac{8 \pi^{\frac{5}{2}}}{\sqrt{3} \gamma} \frac{1}{\alpha^{\prime 2} g_{s}} \\
& \beta=3 \pi^{2} \frac{8 \pi^{\frac{5}{2}}}{\sqrt{3} \gamma} \frac{1}{g_{s}} .
\end{aligned}
$$

Dropping an irrelevant numerical factor, we obtain

$$
S=\frac{1}{\alpha^{\prime 2} g_{s}}\left(-\frac{1}{4} \operatorname{Tr}\left[A_{\mu}, A_{\nu}\right]^{2}-\frac{1}{2} \operatorname{Tr}\left(\bar{\psi} \Gamma^{\mu}\left[A_{\mu}, \psi\right]\right)\right)+3 \pi^{2} \frac{1}{g_{s}} \operatorname{Tr} 1 .
$$

\section{The large-N reduced model of super Yang-Mills the- ory}

\subsection{Double scaling limit}

In this section, we interpret the matrix model (2.22) as an effective theory for the large-N reduced model of ten-dimensional super Yang-Mills theory [4]. It is defined by

$$
S_{0}=\frac{N a^{4}}{g_{0}^{2}}\left(-\frac{1}{4} \operatorname{Tr}\left[A_{\mu}, A_{\nu}\right]^{2}-\frac{1}{2} \operatorname{Tr}\left(\bar{\psi} \Gamma^{\mu}\left[A_{\mu}, \psi\right]\right)\right),
$$

and

$$
-\frac{\pi}{a} \leq \text { eigenvalues of } A_{\mu}<\frac{\pi}{a}
$$

where $a$ is the space time cut-off. In order to find a prescription of the double scaling limit, we consider ten-dimensional super Yang-Mills theory before the large-N reduction:

$$
S_{S Y M}=\frac{N}{g_{0}^{2} a^{6}} \int d^{10} x\left(\frac{1}{4} \operatorname{Tr}\left(F_{\mu \nu} F^{\mu \nu}\right)+\frac{i}{2} \operatorname{Tr}\left(\bar{\psi} \Gamma^{\mu} D_{\mu} \psi\right)\right)
$$


where

$$
\begin{aligned}
F_{\mu \nu} & =\partial_{\mu} A_{\nu}-\partial_{\nu} A_{\mu}+i\left[A_{\mu}, A_{\nu}\right] \\
D_{\mu} \psi & =\partial_{\mu} \psi+i\left[A_{\mu}, \psi\right] .
\end{aligned}
$$

Since $a$ is the cut-off of length and $g_{0}$ is the dimensionless coupling constant, and the theory possesses the classical mass scale $1 / g_{0}^{\frac{1}{3}} a$. The quantization of this model poses some difficulty such as chiral anomaly. However we use this model only to find the mass scale of the large $\mathrm{N}$ reduced model (4.1) and (4.2).

Here we reinterpret the eigenvalues of $A_{\mu}$ as the space-time coordinates. Then $1 / a$ becomes an infrared cut-off and the theory possesses the mass scale $m=g_{0}^{\frac{1}{3}} a$. As we will see in the next subsection, the path integrations of zero modes give rise to the term proportional $\beta$ in (2.22), which we call the chemical potential term, and we can regard the matrix model (2.22) as an effective theory of (4.1) and (4.2). If we assume that we can naively set

$$
\frac{N a^{4}}{g_{0}^{2}}=\alpha=\frac{4 \pi^{\frac{5}{2}}}{\sqrt{6} \gamma} \frac{1}{\alpha^{\prime 2} g_{s}}
$$

and

$$
g_{0}^{2} a^{6}=m^{6}
$$

we obtain

$$
N a^{10} \sim \frac{m^{6}}{\alpha^{\prime 2} g_{s}} .
$$

We see then the double scaling limit should be taken such as

$$
\begin{aligned}
a & \rightarrow 0, \\
g_{0} & \sim a^{-3} \rightarrow \infty, \\
N & \sim a^{-10} \rightarrow \infty .
\end{aligned}
$$

\subsection{Generation of the chemical potential term and one-loop renor- malization}

In this subsection, we consider the quantization of (4.1) and show that the matrix model (2.22) can be regarded as its effective action. In particular we will see that the parameter $\beta$ in (2.22) is understood as a sort of chemical potential of the gas of eigenvalues of $A_{\mu}$ 's. We also examine the one-loop renormalization and discuss that we should take the continuum limit of the reduced model as the nonperturbative definition of the theory we are considering. 
We first consider the path integral for $S_{0}$ (4.1) around a completely diagonal background

$$
p_{\mu}=\left(\begin{array}{cccc}
d_{\mu}^{(1)} & & & \\
& d_{\mu}^{(2)} & & \\
& & \ddots & \\
& & & d_{\mu}^{(N)}
\end{array}\right) .
$$

Here $d_{\mu}^{(i)}$ 's are distributed uniformly from $-l$ to $l$, where $l$ is equal to $\pi / a$ and corresponds to the infrared cut-off when we interpret $A_{\mu}$ 's as the space-time coordinates. Then it is natural to interpret this background as the flat space-time. We denote the partition function for this background as $Z_{0}(N)$. As we have seen in section 3, the integrals of the off-diagonal elements cancel each other between bosons and fermions. On the other hand, the diagonal elements do not appear in the one-loop level, and $Z_{0}(N)$ is formally expressed as

$$
\begin{aligned}
Z_{0}(N) & =\int \prod_{i=1}^{N} \prod_{\mu=0}^{9} \frac{d A_{\mu i i}}{S_{N}} \prod_{\gamma=1}^{16} d \psi_{\gamma i i} d c_{i i} d b_{i i} \\
& =\frac{1}{N !} l^{10 N} \frac{1}{\operatorname{vol}(\text { maximal torus })} \int \prod_{i=1}^{N} \prod_{\gamma=1}^{16} d \psi_{\gamma i i}
\end{aligned}
$$

The zero modes of the ghosts correspond to the maximal torus of the gauge group whose action is trivial on the diagonal background (4.9). Therefore by dimensional analysis, it should be

$$
\int \prod_{i=1}^{N} d c_{i i} d b_{i i}=\frac{1}{\operatorname{vol}(\text { maximal torus })}=\text { const. } \alpha^{\frac{N}{2}} \text {. }
$$

The zero mode integral of $\psi$ acquires non-zero value from higher loop effects. Again by dimensional analysis, we should have

$$
\int \prod_{i=1}^{N} \prod_{\gamma=1}^{16} d \psi_{\gamma i i} \sim \alpha^{2 N}
$$

and finally we obtain

$$
Z_{0}(N)=\operatorname{const} \cdot \frac{1}{N !}\left(l^{10} \alpha^{\frac{5}{2}}\right)^{N}
$$

Next we consider the background

$$
p_{\mu}=\left(\begin{array}{cccc}
\hat{A}_{\mu} & & & \\
& d_{\mu}^{(1)} & & \\
& & \ddots & \\
& & & d_{\mu}^{(N-n)}
\end{array}\right)
$$

Here $\hat{A}_{\mu}$ is a $n \times n$ matrix and $d_{\mu}^{i}$ 's are distributed uniformly from $-l$ to $l$. Denoting the partition function around this background as $Z$, we define the effective action for the $n \times n$ 
block as

$$
S_{\text {eff }}=-\log \frac{Z}{Z_{0}(N)} .
$$

Noting that the contribution from the lower right $(N-n) \times(N-n)$ block is nothing but $Z_{0}(N-n)$ and the contributions from the off-diagonal-blocks are calculated in section 3.4. We obtain

$$
\begin{aligned}
S_{\text {eff }} & =-\log \frac{Z_{0}(N-n)}{Z_{0}(N)}-\alpha \frac{1}{4} \operatorname{Tr}\left[\hat{A}_{\mu}, \hat{A}_{\nu}\right]^{2}+\text { const. } \sum_{i=1}^{N-n} \frac{1}{\left(d^{(i)}\right)^{8}} \operatorname{Tr}(\hat{F} \hat{F} \hat{F} \hat{F})+\cdots \\
& =n \log \left(\frac{l^{10} \alpha^{\frac{5}{2}}}{N}\right)-\alpha \frac{1}{4} \operatorname{Tr}\left[\hat{A}_{\mu}, \hat{A}_{\nu}\right]^{2}+\text { const. } \frac{N}{l^{10}} l^{2} \operatorname{Tr}(\hat{F} \hat{F} \hat{F} \hat{F})+\cdots,
\end{aligned}
$$

where $\hat{F} \sim i\left[\hat{A}_{\mu}, \hat{A}_{\nu}\right]$. As was shown in the previous subsection, in the double scaling limit, $N \sim l^{10}$. Therefore we have

$$
S_{\text {eff }}=\text { const. } n-\alpha \frac{1}{4} \operatorname{Tr}\left[\hat{A}_{\mu}, \hat{A}_{\nu}\right]^{2}+\text { const. } \frac{1}{a^{2}} \operatorname{Tr}(\hat{F} \hat{F} \hat{F} \hat{F})+\cdots .
$$

The first term is the chemical potential term we are looking for. The third term and the following terms are cut-off dependent and divergent. This means that we have to add counter terms to $S_{0}$ (4.1). As is well-known in the large-N gauge theory, the structures of these divergences are the same as those of the original ten-dimensional super Yang-Mills theory (4.3) [4], which is not renormalizable perturbatively. We cannot give a complete definition of the theory (4.1) only by the perturbation theory as we cannot do so for the super Yang-Mills theory (1.3). Namely we expect that there is a nontrivial fixed point of renormalization group of the theory (4.1) and we can take the continuum limit around it to define the theory nonperturbatively. The divergences of the loop corrections are mild due to supersymmetry, which supports the existence of the nontrivial fixed point. At the one loop level the degree of the divergences is less than or equal to two. There would be the sixth order divergences without supersymmetry. We also expect that the matrix model we consider is controlled by a universality similar to field theory. 


\subsection{Moduli space of the matrix model}

Our matrix model possesses a large number of degenerate vacua at least perturbatively. In this subsection, we consider the following classical solution:

$$
\begin{aligned}
& \bar{A}_{0}=\left(\begin{array}{llll}
d_{0}^{(1)} & & & \\
& d_{0}^{(2)} & & \\
& & \ddots & \\
& & & d_{0}^{(N)}
\end{array}\right), \\
& \bar{A}_{i}=0,
\end{aligned}
$$

where $d_{0}^{(i)}$ 's are distributed uniformly from 0 to $T$. At this point of the moduli space, our action (4.1) becomes the following in the $A_{0}=\overline{A_{0}}$ gauge:

$$
\int_{0}^{T} d \tau \frac{1}{2}\left[-\operatorname{Tr}\left(\frac{\partial A_{i}}{\partial \tau}\right)^{2}-\frac{1}{2} \operatorname{Tr}\left[A_{i}, A_{j}\right]^{2}-i \operatorname{Tr} \psi \partial_{\tau} \psi-\operatorname{Tr} \bar{\psi} \Gamma^{i}\left[A_{i}, \psi\right]\right] .
$$

Here we have put $\left[\bar{A}_{0}, A_{i}\right]=i \partial_{\tau} A_{i},\left[\bar{A}_{0}, \psi\right]=i \partial_{\tau} \psi$ which can be justified in the large-N limit [4]. After the Wick rotation $(\tau \rightarrow i t)$, this action can be seen to coincide with that of Banks, Fischler, Shenker and Susskind [5]:

$$
\frac{1}{2 g}\left[\operatorname{Tr} \dot{X}^{i} \dot{X}^{i}+2 \operatorname{Tr} \bar{\theta} \gamma_{-} \dot{\theta}-\frac{1}{2} \operatorname{Tr}\left[X^{i}, X^{j}\right]^{2}-2 \operatorname{Tr} \bar{\theta} \gamma_{-} \gamma^{i}\left[\theta, X^{i}\right]\right]
$$

The fermionic variables are 32 component eleven-dimensional spinors, satisfying the lightcone constraint $\gamma_{+} \theta=0$. They have proposed this action as a nonperturbative formulation of M-theory in the light-cone frame. Therefore we found that our matrix model contains type IIA string theory as well!

However we have always regard our matrix model as a nonperturbative definition of type IIB string theory in this paper. In section 3, we have identified particular moduli which can be interpreted in terms of type IIB string theory. However these findings are not mutually contradictory since type IIA and type IIB string theories are T-dual to each other.

Therefore we can view the finding of this section as another manifestation of duality. Namely the different points of the moduli space of our matrix model may be interpreted either by type IIB string theory or by type IIA string theory. This feature is also very reminiscent of $N=2$ supersymmetric gauge theories. 


\section{Summary and discussion}

In this paper, we proposed a matrix model which may give a constructive definition of string theory. We showed that the matrix model is connected to the type IIB GreenSchwarz action and possesses the $N=2$ supersymmetry, which is a sufficient condition for the theory to include gravitation, and that the space-time is dynamically generated via the collective coordinate of this model. We also showed that the massless spectrum is consistent with string theory by examining the interactions between the BPS-saturated states. We conjectured that the theory can be defined nonperturbatively by the continuum limit of the large- $\mathrm{N}$ reduced model of ten-dimensional super Yang-Mills theory, whose existence is expected due to the supersymmetry, and pointed out that the marginal preservation of the $U(1)^{d}$ symmetry is important for the generation of space time.

Finally we discuss the relation between the Wilson loop and the classical solutions for $A_{\mu}$ 's. The Wilson loop of the large-N reduced model is given by

$$
W[\xi(\sigma)]=\operatorname{Tr} P \exp \left(i \oint_{0}^{2 \pi} A_{\mu} \dot{\xi}^{\mu}(\sigma) d \sigma\right)
$$

where $\xi^{\mu}(\sigma)$ satisfies

$$
\xi^{\mu}(2 \pi)=\xi^{\mu}(0)+n^{\mu} a
$$

and the $n^{\mu}$ 's are integers and correspond to the winding numbers. As in the previous sections if we regard $A_{\mu}$ 's as the space-time coordinates, $\dot{\xi}^{\mu}$ corresponds to the momentum. Therefore it is natural to consider the Wilson loop (5.1) as the creation operator of the string state with momentum eigenvalues $\left|k^{\mu}(\sigma)=\dot{\xi}^{\mu}(\sigma)\right\rangle$. If we identify the Wilson loops with strings, then it is natural to identify the classical solutions for $A_{\mu}$ 's with D-objects. In order to see that it is really the case, we again consider the static D-string solution:

$$
\begin{aligned}
& p_{0}^{D}=\left(\begin{array}{llll}
\frac{T}{\sqrt{2 \pi n}} q & & & \\
& d_{0}^{(1)} & & \\
& & \ddots & \\
& & & d_{0}^{(N-n)}
\end{array}\right), \\
& p_{1}^{D}=\left(\begin{array}{llll}
\frac{L}{\sqrt{2 \pi n}} p & & & \\
& d_{1}^{(1)} & & \\
& & \ddots & \\
& & & d_{1}^{(N-n)}
\end{array}\right),
\end{aligned}
$$




$$
p_{i}^{D}=\left(\begin{array}{llll}
a_{i} 1_{n} & & & \\
& d_{i}^{(1)} & & \\
& & \ddots & \\
& & & d_{i}^{(N-n)}
\end{array}\right)
$$

Here the first block represents a D-string parallel to the $X^{0}-X^{1}$ plane and located at $X^{i}=a^{i}$, and the rest represents the flat vacuum. Therefore $p_{\mu}^{D}$ represents one D-string sitting in the vacuum, while the flat vacuum is expressed as

$$
p_{\mu}^{v a c}=\left(\begin{array}{cccc}
d_{\mu}^{(1)} & & & \\
& d_{\mu}^{(2)} & & \\
& & \ddots & \\
& & & d_{\mu}^{(N)}
\end{array}\right) .
$$

The difference of the Wilson loop for these backgrounds is given in the tree level by

$$
<W\left(k^{\mu}(\sigma)\right)>_{A_{\mu}=p_{\mu}^{D}}-<W\left(k^{\mu}(\sigma)\right)>_{A_{\mu}=p_{\mu}^{v a c}}=e^{i k_{0}^{i} a^{i}} \operatorname{Tr} P \exp \left[i \oint\left(\frac{T}{2 \pi n} q k^{0}(\sigma)+\frac{L}{2 \pi n} p k^{1}(\sigma)\right) d \sigma\right] .
$$

If the Wilson loops correspond to strings and the classical solutions correspond to D-strings, this quantity should be compared with the closed string tadpole wave function in the presence of the D-string. Denoting the boundary state corresponding to the D-string as $|D\rangle$, the closed tadpole wave function is given by

$$
\langle k(\sigma) \mid D\rangle=\delta\left(k^{0}(\sigma)\right) \delta\left(k^{1}(\sigma)\right) e^{i k_{0}^{i} a^{i}}
$$

The exponential factor representing the Dirichlet boundary condition in (5.6) is reproduced in (5.5). Since the Wilson loop does not create an on-shell state, we cannot demand the exact coincidence. At any rate the interpretation of the Wilson loops and D-strings seems consistent. This is the reason why we have called the classical solutions as D-strings.

We would like to thank H. Aoki, K.-J. Hamada, T. Tada and K. Yoshida for discussions and J.H. Schwarz for valuable comments.

\section{Note added}

After finishing this paper, we have been informed that Jevicki and Yoneya [10], and Periwal [11] have considered related models. 


\section{Appendix}

In this appendix, we calculate the one-loop quantum corrections of the matrix models (2.21) and (4.1). As in the ordinary background field method in the quantum field theories, we decompose the matrices $A_{\mu}$ and $\psi$ to the backgrounds and the quantum fluctuations. Namely,

$$
\begin{aligned}
A_{\mu} & =p_{\mu}+a_{\mu}, \\
\psi & =\chi+\varphi
\end{aligned}
$$

where $p_{\mu}$ and $\chi$ are backgrounds and $a_{\mu}$ and $\varphi$ are quantum fluctuations. The action is expanded up to the second order of the quantum fluctuations such as

$$
\begin{aligned}
S_{2}= & -\operatorname{Tr}\left(\frac{1}{4}\left[p_{\mu}, p_{\nu}\right]^{2}+\frac{1}{2} \bar{\chi} \Gamma^{\mu}\left[p_{\mu}, \chi\right]\right. \\
& -a_{\nu}\left(\left[p_{\mu},\left[p_{\mu}, p_{\nu}\right]\right]+\frac{1}{2}\left[\bar{\chi} \Gamma^{\mu}, \chi\right]\right)+\bar{\psi} \Gamma^{\mu}\left[p_{\mu}, \varphi\right] \\
& +\frac{1}{2}\left[p_{\mu}, a_{\nu}\right]^{2}-\frac{1}{2}\left[p_{\mu}, a_{\mu}\right]^{2}+\left[p_{\mu}, p_{\nu}\right]\left[a_{\mu}, a_{\nu}\right] \\
& \left.+\frac{1}{2} \bar{\varphi} \Gamma^{\mu}\left[p_{\mu}, \varphi\right]+\bar{\chi} \Gamma^{\mu}\left[a_{\mu}, \varphi\right]\right),
\end{aligned}
$$

where the terms in the second line vanishes due to the equations of motions, or are dropped in the back ground field method. To fix the gauge invariance

$$
\begin{aligned}
\delta A_{\mu} & =i\left[A_{\mu}, \alpha\right], \\
\delta \psi & =i[\psi, \alpha],
\end{aligned}
$$

we add the gauge fixing term

$$
S_{g . f .}=-\operatorname{Tr}\left(\frac{1}{2}\left[p_{\mu}, a_{\mu}\right]^{2}+\left[p_{\mu}, b\right]\left[p_{\mu}, c\right]\right),
$$

where $c$ and $b$ are ghosts and anti-ghosts, respectively. In the following, we set $\chi=0$. Dropping the first order term of the quantum fluctuations, we obtain

$$
\begin{aligned}
\tilde{S}_{2}=S_{2}+S_{g . f}=-T r & \left(\frac{1}{2}\left[p_{\mu}, a_{\nu}\right]^{2}+\left[p_{\mu}, p_{\nu}\right]\left[a_{\mu}, a_{\nu}\right]\right. \\
& \left.+\frac{1}{2} \bar{\varphi} \Gamma^{\mu}\left[p_{\mu}, \varphi\right]+\left[p_{\mu}, b\right]\left[p_{\mu}, c\right]\right) .
\end{aligned}
$$

Here we introduce a notation of adjoint operators. For an operator $o$,

$$
[o, X]=O X .
$$


Using this, we rewrite (A.12) as

$$
\tilde{S}_{2}=\operatorname{Tr}\left(\frac{1}{2} a_{\mu}\left(P_{\lambda}^{2} \delta_{\mu \nu}-2 i F_{\mu \nu}\right) a_{\nu}-\frac{1}{2} \bar{\varphi} \Gamma^{\mu} P_{\mu} \varphi+b P_{\lambda}^{2} c\right),
$$

where

$$
\begin{aligned}
{\left[p_{\mu}, X\right] } & =P_{\mu} X \\
{\left[f_{\mu \nu}, X\right] } & =F_{\mu \nu} X, f_{\mu \nu}=i\left[p_{\mu}, p_{\nu}\right] .
\end{aligned}
$$

From this, the one-loop effective action $W$ is evaluated as

$$
\begin{aligned}
W= & -\log \int d a d \varphi d c d b e^{-\tilde{S}_{2}} \\
= & \frac{1}{2} \operatorname{Tr} \log \left(P_{\lambda}^{2} \delta_{\mu \nu}-2 i F_{\mu \nu}\right)-\frac{1}{4} \operatorname{Tr} \log \left(\left(P_{\lambda}^{2}+\frac{i}{2} F_{\mu \nu} \Gamma^{\mu \nu}\right)\left(\frac{1+\Gamma_{11}}{2}\right)\right) \\
& -\operatorname{Tr} \log \left(P_{\lambda}^{2}\right)+i \theta,
\end{aligned}
$$

where $\theta$ is the anomaly term. We can show that $\theta$ vanishes for the two cases we have studied in this paper.

$$
\text { Case(1): } F_{\mu \nu}=0 .
$$

This is because we can simultaneously diagonalize $P_{\mu}$ 's and $\Gamma^{\mu} P_{\mu}$ becomes a real operator.

$$
\text { Case(2): } P_{i}=0 \text { for at least in one direction } i \text {. }
$$

This is because

$$
\operatorname{det}\left(\Gamma^{\mu} P_{\mu}\right)^{\dagger}\left(\Gamma^{\nu} P_{\nu}\right)=\operatorname{det}\left(\Gamma^{i} \Gamma^{\mu} P_{\mu}\right)^{2} .
$$




\section{References}

[1] A. Sen, Int. J. Mod. Phys. A9 (1994) 3703;

J.H. Schwarz, Lett. Math. Phys. 34 (1995) 309;

J. Maharana and J. Schwarz, Nucl. Phys. B390 (1993) 3. th

[2] E. Witten, Nucl. Phys. 443 (1995) 85.

[3] J. Polchinski, Phys. Rev. Lett. 75 (1995) 4724.

[4] T. Eguchi and H. Kawai, Phys. Rev. Lett. 48 (1982) 1063.

G. Parisi, Phys. Lett. 112B (1982) 463.

D. Gross and Y. Kitazawa, Nucl. Phys. B206 (1982) 440.

G. Bhanot, U. Heller and H. Neuberger, Phys. Lett. 113B (1982) 47.

S. Das and S. Wadia, Phys. Lett. 117B (1982) 228.

[5] T. Banks, W. Fischler, S.H. Shenker and L. Susskind, hep-th/9610043.

[6] M. Green and J. Schwarz, Phys. Lett. 136B (1984) 367.

[7] A. Schild, Phys. Rev. D16 (1977) 1722.

[8] The similarity between the large- $\mathrm{N}$ reduced model and string theory in the Schild gauge was investigated by Bars some time ago for bosonic string case: I. Bars, Phys. Lett. 245B(1990)35; 'Strings 90', eds. R. Arnowitt et. al. (Proc. of the Superstring Workshop, Texas A \& M Univ., 1990) p.202; 'Beyond the Standard Model', ed. K. Milton (Proc. of conference in Oklahoma) p.72.

[9] E. Witten and D. Olive, Phys. Lett. 78B (1978) 97.

[10] A. Jevicki and T. Yoneya, private communication.

[11] V. Periwal, hep-th/9611103. 